\title{
The future of organ-oriented research and treatment
}

\author{
Qiang Zhao", Yu Nie", Zhiyong Guo, Xiaoshun He \\ Organ Transplant Center, The First Affiliated Hospital, Sun Yat-sen University, Guangzhou 510080, China \\ \#These authors contributed equally to this work. \\ Correspondence to: Xiaoshun He. Organ Transplant Center, The First Affiliated Hospital, Sun Yat-sen University, Number 58 Zhongshan Er Road, \\ Guangzhou 510080, China. Email: gdtrc@163.com.
}

Submitted Aug 13, 2019. Accepted for publication Aug 20, 2019.

doi: $10.21037 /$ hbsn.2019.08.09

View this article at: http://dx.doi.org/10.21037/hbsn.2019.08.09

Organ-oriented research platform is based on machine perfusion (MP) technology providing continuous oxygen and nutrient supply for harvested organs. It permits in vivo and in vitro investigation and represents one of the latest innovations in the quest for a model to explore either physiological or pathological processes of the whole organ. Recently, the ex vivo animal perfusion model has proven to be an effective and reproducible translational research platform (1). Preliminary clinical studies have demonstrated MP's a new promising platform that allows pre-transplant $e x$ vivo preservation, functional assessment, reconditioning for donor grafts, bringing increasing graft utilization and improving transplantation outcomes (2). With MP, we have even successfully established an innovative technology called ischemia-free organ transplantation platform and completed human's first case of ischemia-free liver transplantation (3).

Based on recent remarkable research results using MP in solid organ transplantation, there has been growing interest in and expectations for the application of MP technology on organ level research beyond transplantation. We believe through combining with advances in surgery, oncology, pharmacology, immunology, metabolomics, gene and (stem) cell therapy, the MP technology has great potential and opportunity to establish an organ level research and treatment platform for human medicine and diseases.

\section{Multivisceral preservation system}

The first step to establish organ-oriented research platform is to overcome the challenge of ex vivo multiorgan preservation. Our team successfully developed a "multivisceral preservation system" (Life-X) based on MP in 2016 (4). As is shown in Figure 1, Life-X can mimic the circulatory system of human body to provide blood and nutrition to maintain the isolated organs (the liver and kidney for example) in vitro in constant circulation. Our newest study has proved the validity of Life- $X$ in preserving multiple organs in vitro with real-time, accurate function evaluation and even repairing the damaged graft functions from cold and warm ischemic injury (4).

With Life-X, the researchers can not only preserve and cultivate the organs in vitro in the simulative condition of homeostasis, but can also administer medications and supplements to the target organs for research while monitoring and evaluating organ functions at the same time, which guaranteed a platform for single and multiple organ level research. By combining with advances in relative subjects, there will be tremendous therapeutic potential for Life-X system to advance organ level study and its future translation into establishing organ-oriented research and treatment platform.

\section{In vitro organ culture and accurate function evaluation}

The organ culture system will bring us the future of accurate functional evaluation and precise intervention for specific organs. The in vitro liver function evaluation during MP has been successfully achieved in clinical practices (2). An intestinal organ culture system has uncovered roles for nervous system in microbe-immune crosstalk (5). In an exciting study by Vrselja et al., the disembodied brains of pigs could be even restored and maintained outside body up to $4 \mathrm{~h}$ with real-time metabolism and neurological function assessment under perfusion system, demonstrating the possibility of the isolated, intact organ culture in vitro following with accurate organ function study on microcirculation and molecular/cellular activity (6). 

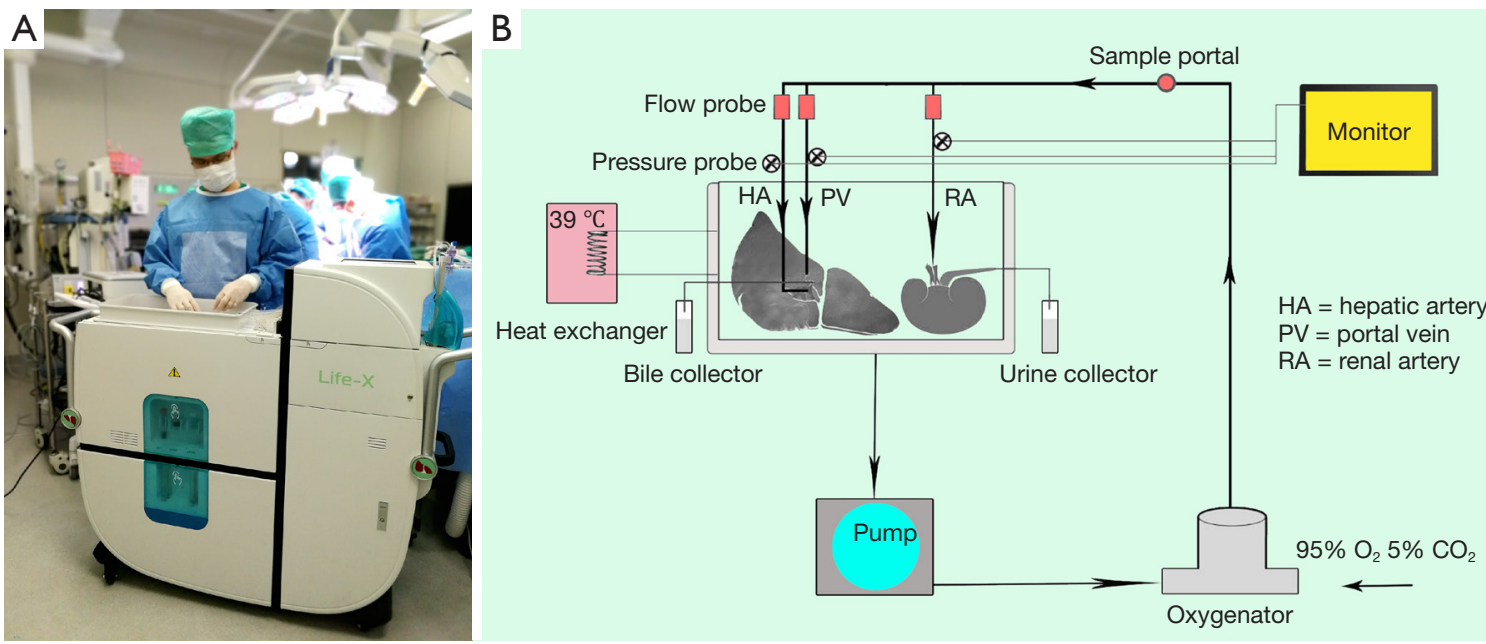

Figure 1 Schematic diagram of Multivisceral Repairing System (Life-X) and circuit. (A) Life-X device; (B) scheme of the Life-X circuit. The perfusion system was composed of an organ chamber, a roller pump, an oxygenator, and a heat exchanger. All of these compartments were connected by tubing. Infusion pumps were connected to ports along the tubing to infuse medications and supplements. Sampling ports are present in both the afferent and efferent arms of the circuits perfusing the liver and kidney. The perfusion parameters, physiological status and functional changes of the isolated organs were recorded in real-time.

Under the in vitro Life-X organ research platform, perfusates formulation or medications could be changed combined with metabolomics analysis to explore the precise organ metabonomics. By achieving a comprehensive, realtime and accurate assessment of organ function, it's hopeful to find out new biomarkers or even establish comprehensive scoring systems for organ function evaluation or predicting post-transplant outcomes (7). Moreover, the precise mechanisms of organ function at cell/molecular level could be further investigated by combining optical imaging technology, providing us new methods for the disease diagnosis and treatment (8).

\section{Organ pharmacology and toxicology research platform}

Due to the complexity of human body, the current in vitro evaluation models and animal experiments are insufficient to accurately reflect drug's pharmic effects. Using organoriented research platform, accurate pharmacology and pharmacodynamic evaluation could be conducted on specific isolated organs. Isolated organ perfusion (IOP) has helped to illustrate the mechanism of physiologicallybased and pharmacokinetics-based hepatic clearance of chemical-drug interactions between xenobiotics (9). Isolated hepatic perfusion model has also turn out to possess stable pharmacokinetic effects, limited toxicity and excellent response on chemotherapeutics study with unresectable liver metastasis. This organ-oriented evaluation system, that is more practical and drup-efficient, could effectively be used as a platform for physiological research and drug development. Using human discarded organs combined with IOP technique, the Life-X system could be effectively used to establish organ level research platform for drug pharmacodynamics and toxicity study. This platform can better mimic the real response of human body to drugs and chemicals while significantly reduce the cost and time of toxicity assessment, making it the more accurate toxicity evaluating methods and promising front field of toxicity evaluation in the future.

\section{In vitro diseased organ research platform}

An additional advantage of Life-X system is the opportunity to establish effective in vitro diseased organ models of human disease using diseased liver, kidney and other diseased organs after surgical resection, which will help to investigate the mechanisms of disease and identify possible treatments. As a proof of concept, a human discarded liver was preserved in vitro up to 86 hours using MP (10). Considering the possibility of long time ex vivo organ preservation, establishing an in vitro diseased organ model like liver cirrhosis, hepatocellular carcinoma or steatosis hepatis using diseased livers from recipients becomes possible. Boteon and colleagues successfully constructed a 


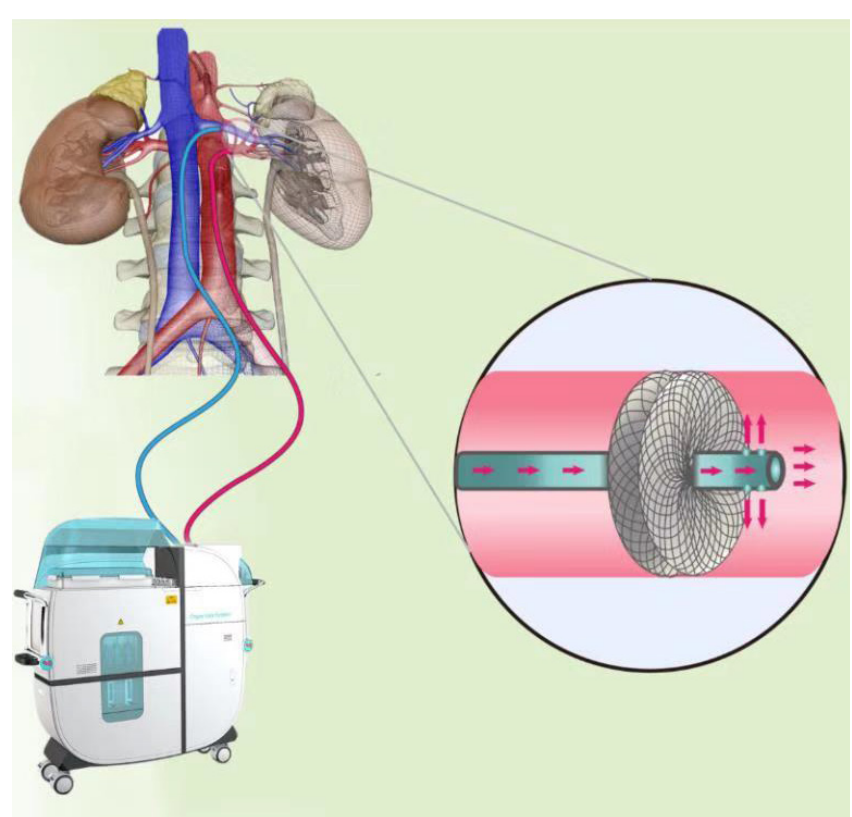

Figure 2 Schematic diagram of organ arterial and venous occlusion and blood perfusion.

steatosis model using discarded steatotic livers, following with successful manipulation of lipid metabolism by defatting therapies during MP (11). Through setting up diseased lung model using discarded lung infected by hepatitis $\mathrm{C}$ virus (HCV), Galasso et al. have explored out a method for treatment of $\mathrm{HCV}$-infected donor lungs that prevents HCV transmission (12). These studies have taken the first steps toward constructing in vitro diseased organ research platform and the corresponding results are encouraging. Further studies are warranted to construct reliable diseased organ models, including tumors, infectious diseases (viral hepatitis, cirrhosis, etc.), chronic diseases (chronic obstructive pulmonary disease) and metabolic diseases [non-alcoholic fatty liver disease (NAFLD)].

\section{Gene and stem-cell therapy research platform}

Similar to medication therapy, gene therapy by administering small interfering RNA (siRNA) or adenovirus during MP is also a feasible, promising approach to investigate or treat the targeted organs. Pilot studies of adenoviral interleukin-10 gene transfection during MP were reported with notable effects and improved posttransplant outcomes. Breakthroughs on non-viral gene delivery methods like nanoparticles, liposome, and oligonucleotide) during MP have also been achieved. Recently, siRNA targeting liver was successfully transfected during MP and proved to attenuate MHC-II expression in vascular endothelial cells for 4-6 weeks after transplantation, showing the potential of organ translational research and transformational therapeutic applications (13). These encouraging studies foreshadow the potential major impact of gene therapy under MP based organ research platform. It is equally true that under Life-X platform, gene interference therapeutics targeting specific or multivisceral organs can be achieved, making it an exciting frontier with promising transformational potential to improve clinical outcomes.

The combination of stem-cell therapy and ex vivo organ perfusion is a promising technique and has also become a potential clinical reality for certain organ diseases. Experimental researches and clinical trials testing the administration of immune cells or mesenchymal stem cells (MSCs) during MP have explored the therapeutic potential of cell therapy. Immune modulation could be performed by isolating liver and perfusing with nT-regs, showing reduced host adaptive immune responses and improved allograft survival (14). Transplanting MSCs during MP have proved its ability to effectively reduce the immune response and inflammation in donor graft during I/R injury (15). By delivering the specific stem cells or cell subsets, this promising technique may help to elucidate the characteristics of specific immune cell subsets in organs and finally discover the mechanisms of specific organ diseases.

\section{Organ-oriented treatment platform}

The development of most diseases is usually restricted to one independent organ. Nowadays, there is still lack of precise medical treatment for one targeted organ. Based on Life-X, we propose a new concept of organ-oriented treatment platform that integrates interventation technique, radiology and medication treatment (Figure 2). Under the guidance and surveillance of radiography, the input and output vessels of the organ will be blocked by interventional technique, while the blood circulation of the organ will be maintained by Life-X. Thus, a certain organ can be temporarily disconnected from human body while its blood flow is temporally taken over by Life-X. Then the precise drug administration for the isolated organ can be achieved. Compared with traditional systemic treatment, organoriented treatment provides the advantages that the drugs can directly and continuously target on the lesion through circulation in the isolated organ. Then it can increase the drug concentration in the lesion, maximize the curative 
effect, and diminish the occurrence of systemic side-effects. With the advantages of minimal invasion, accuracy, high efficiency and limited toxicity, this platform may become the preferred treatment method for certain diseases restricted in one solid organ in the future.

\section{Conclusions}

The MP platform like Life-X system can maintain isolated organs in vitro in functional homeostasis, promising to be a major breakthrough for establishing effective organ-oriented research and treatment platform, especially in translational organ transplant research. These platforms have tremendous potential and broad application prospects when combining with other advances in surgery, pathology, pharmacy, oncology, and bioengineering. Future pre-clinical studies are needed to explore the validity and reliability of this organ-oriented research and treatment platform based on MP technique.

\section{Acknowledgments}

None.

\section{Footnote}

Conflicts of Interest: The authors have no conflicts of interest to declare.

Ethical Statement: The authors are accountable for all aspects of the work in ensuring that questions related to the accuracy or integrity of any part of the work are appropriately investigated and resolved.

\section{References}

1. Kumar R, Chung WY, Dennison AR, et al. Ex Vivo Porcine Organ Perfusion Models as a Suitable Platform for Translational Transplant Research. Artif Organs 2017;41:E69-79.

2. Nasralla D, Coussios CC, Mergental H, et al. A randomized trial of normothermic preservation in liver transplantation. Nature 2018;557:50-6.

3. He X, Guo Z, Zhao Q, et al. The first case of ischemiafree organ transplantation in humans: A proof of concept. Am J Transplant 2018;18:737-44.

4. He X, Ji F, Zhang Z, et al. Combined liver-kidney perfusion enhances protective effects of normothermic perfusion on liver grafts from donation after cardiac death. Liver Transpl 2018;24:67-79.
5. Yissachar N, Zhou Y, Ung L, et al. An Intestinal Organ Culture System Uncovers a Role for the Nervous System in Microbe-Immune Crosstalk. Cell 2017;168:1135-48.e12.

6. Vrselja Z, Daniele SG, Silbereis J, et al. Restoration of brain circulation and cellular functions hours postmortem. Nature 2019;568:336-43.

7. Mergental H, Stephenson BTF, Laing RW, et al. Development of Clinical Criteria for Functional Assessment to Predict Primary Nonfunction of High-Risk Livers Using Normothermic Machine Perfusion. Liver Transpl 2018;24:1453-69.

8. Liu Q, Nassar A, Buccini L, et al. Lipid metabolism and functional assessment of discarded human livers with steatosis undergoing 24 hours of normothermic machine perfusion. Liver Transpl 2018;24:233-45.

9. Poulin P, Bteich M, Haddad S. Supplemental Analysis of the Prediction of Hepatic Clearance of Binary Mixtures of Bisphenol A and Naproxen Determined in an Isolated Perfused Rat Liver Model to Promote the Understanding of Potential Albumin-Facilitated Hepatic Uptake Mechanism. J Pharm Sci 2017;106:3207-14.

10. Liu Q, Nassar A, Buccini L, et al. Ex situ 86-hour liver perfusion: Pushing the boundary of organ preservation. Liver Transpl 2018;24:557-61.

11. Boteon YL, Boteon APCS, Attard J, et al. Ex situ machine perfusion as a tool to recondition steatotic donor livers: Troublesome features of fatty livers and the role of defatting therapies. A systematic review. Am J Transplant 2018;18:2384-99.

12. Galasso M, Feld JJ, Watanabe Y, et al. Inactivating hepatitis $\mathrm{C}$ virus in donor lungs using light therapies during normothermic ex vivo lung perfusion. Nat Commun 2019;10:481.

13. Tietjen GT, Hosgood SA, DiRito J, et al. Nanoparticle targeting to the endothelium during normothermic machine perfusion of human kidneys. Sci Transl Med 2017. doi: 10.1126/scitranslmed.aam6764.

14. Mohamed MS. Combined Ex Vivo Organ Perfusion and Mesenchymal Stem Cells Transplantation. J Stem Cells 2016;11:213-7.

15. Schlegel A, Kron P, Graf R, et al. Hypothermic Oxygenated Perfusion (HOPE) downregulates the immune response in a rat model of liver transplantation. Ann Surg 2014;260:931-7; discussion 937-8.

Cite this article as: Zhao Q, Nie Y, Guo Z, He X. The future of organ-oriented research and treatment. HepatoBiliary Surg Nutr 2019;8(5):502-505. doi: 10.21037/hbsn.2019.08.09 